\title{
Precise Asymptotics on Second-Order Complete Moment Convergence of Uniform Empirical Process
}

\author{
Junshan $\mathrm{Xie}^{1}$ and Lin $\mathrm{He}^{2}$ \\ ${ }^{1}$ College of Mathematics and Information, Henan University, Kaifeng 475000, China \\ ${ }^{2}$ Department of Economics, Zhengzhou Institute of Finance and Economics, Zhengzhou 450000, China
}

Correspondence should be addressed to Junshan Xie; junshan@henu.edu.cn

Received 10 May 2014; Accepted 21 July 2014; Published 27 August 2014

Academic Editor: Ahmed El-Sayed

Copyright (C) 2014 J. Xie and L. He. This is an open access article distributed under the Creative Commons Attribution License, which permits unrestricted use, distribution, and reproduction in any medium, provided the original work is properly cited.

Let $\left\{\xi_{i}, 1 \leq i \leq n\right\}$ be a sequence of iid $U[0,1]$-distributed random variables, and define the uniform empirical process $F_{n}(t)=$ $n^{-1 / 2} \sum_{i=1}^{n}\left(I_{\left\{\xi_{i} \leq t\right\}}-t\right), 0 \leq t \leq 1,\left\|F_{n}\right\|=\sup _{0 \leq t \leq 1}\left|F_{n}(t)\right|$. When the nonnegative function $g(x)$ satisfies some regular monotone conditions, it proves that $\lim _{\epsilon \searrow 0}(1 /-\log \epsilon) \sum_{n=1}^{\infty}\left(g^{\prime}(n) / g(n)\right) \mathbb{E}\left\{\left\|F_{n}\right\|^{2} I_{\left\{\left\|F_{n}\right\| \geq \epsilon \sqrt{g(n)}\right\}}\right\}=\pi^{2} / 6$.

\section{Introduction and Main Result}

Let $\left\{X, X_{n}, n \geq 1\right\}$ be a sequence of iid random variables and $S_{n}=\sum_{i=1}^{n} X_{i}$. Hsu and Robbins [1] introduced the concept of complete convergence and obtained that $\sum_{n=1}^{\infty} P\left(\left|S_{n}\right| \geq \epsilon n\right)<$ $\infty, \epsilon>0$, whenever $\mathbb{E} X=0$ and $\mathbb{E} X^{2}<\infty$. The result is extended by Baum and Katz [2], who obtained that, for $0<$ $p<2, r \geq p, \sum_{n=1}^{\infty} n^{(r / p)-2} P\left(\left|S_{n}\right| \geq \epsilon n^{1 / p}\right)<\infty, \epsilon>0$, if and only if $\mathbb{E}|X|^{r}<\infty$, and when $r \geq 1, \mathbb{E} X=0$. Since then, some researchers concern the convergence of the series

$$
\sum_{n=1}^{\infty} \varphi(n) P\left(\left|S_{n}\right| \geq \epsilon f(n)\right), \quad \epsilon>0,
$$

where $\varphi(n)$ and $f(n)$ are all positive functions defined on $[1, \infty)$, and $\sum_{n=1}^{\infty} \varphi(n)=\infty$. Because of the fact that the series tends to infinity when $\epsilon \searrow 0$, one of the interesting problems is to examine the rate when it occurs; then we need to find a suitable normalizing rate function $\psi(\epsilon)$ such that it, multiplied by the series, has a nontrivial limit. The research on this topic is usually called "precise asymptotics." Heyde [3] first proved that

$$
\lim _{\epsilon \searrow 0} \epsilon^{2} \sum_{n=1}^{\infty} P\left(\left|S_{n}\right| \geq \epsilon n\right)=\mathbb{E} X^{2}
$$

whenever $\mathbb{E} X=0$ and $\mathbb{E} X^{2}<\infty$. Chow [4] studied the similar result on complete moment convergence of $\sum_{n=1}^{\infty} n^{p \alpha-2-\alpha} \mathbb{E}\left\{\max _{1 \leq j \leq n}\left|S_{j}\right|-\epsilon n^{\alpha}\right\}_{+}$. Later, Liu and Lin [5] extended the result to the $p(p<2)$ order complete moment convergence, which states that when $\mathbb{E} X=0, \mathbb{E} X^{2}=\sigma^{2}$, and $\mathbb{E} X^{2} \log ^{+}|X|<\infty$, then

$$
\lim _{\epsilon \searrow 0} \frac{1}{-\log \epsilon} \sum_{n=1}^{\infty} \frac{1}{n^{2}} \mathbb{E}\left|S_{n}\right|^{2} I_{\left\{\left|S_{n}\right| \geq \epsilon n\right\}}=2 \sigma^{2} .
$$

In addition to the partial sums of iid random variables, there are some corresponding precise asymptotic results on other subjects, such as uniform empirical process, selfnormalized sums, order statistics, eigenvalue statistics, and random fields. For the details on this topic, one can refer to Gut and Steinebach [6].

The paper will focus on the precise asymptotic of the uniform empirical process. Let $\left\{\xi_{i}, 1 \leq i \leq n\right\}$ be a sequence of independent $U[0,1]$-distributed random variables; we can define the uniform empirical process $F_{n}(t)=$ $n^{-1 / 2} \sum_{i=1}^{n}\left(I_{\left\{\xi_{i} \leq t\right\}}-t\right), 0 \leq t \leq 1,\left\|F_{n}\right\|=\sup _{0 \leq t \leq 1}\left|F_{n}(t)\right|$. Consider $B$ to be a Brownian bridge on $D[0,1]$ and write $\|B\|=\sup _{0 \leq t \leq 1}|B(t)|$. Zhang and Yang [7] established some precise asymptotics on the complete convergence of the uniform empirical process; one of their main results can be stated as follows. 
Lemma 1. For $1 \leq p<2, r>p$, then

$$
\begin{aligned}
\lim _{\epsilon \searrow 0} \epsilon^{2(r-p) /(2-p)} \sum_{n=1}^{\infty} n^{(r / p)-2} P\left(\left\|F_{n}\right\| \geq \epsilon n^{(1 / p)-(1 / 2)}\right) \\
=\frac{p}{r-p} \mathbb{E}\|B\|^{2(r-p) /(2-p)} .
\end{aligned}
$$

Zang and Huang [8] obtained some results on the first-order complete moment convergence of $F_{n}(t)$. If the nonnegative function $g(x)$ satisfies some regular monotone conditions, they proved the following.

Lemma 2. For any $s>0$, one has

$$
\lim _{\epsilon \searrow 0} \frac{1}{-\log \epsilon} \sum_{n=1}^{\infty} \frac{g^{\prime}(n)}{g(n)} \mathbb{E}\left\{\left\|F_{n}\right\|-\epsilon g^{s}(n)\right\}=\frac{1}{s} \mathbb{E}\|B\| .
$$

Chen and Zhang [9] further got some precise asymptotic result on the second-order complete moment convergence of it. A typical result in their work can be listed as follows.

Lemma 3. For any $0<\beta \leq 2, \delta>(2 / \beta)-1$, one has

$$
\begin{aligned}
\lim _{\epsilon \searrow 0} \epsilon^{\beta(\delta+1)-2} \sum_{n=1}^{\infty} \frac{(\log n)^{\delta-(2 / \beta)}}{n} \mathbb{E}\left\{\left\|F_{n}\right\|^{2} I_{\left\{\left\|F_{n}\right\| \geq \epsilon(\log n)^{1 / \beta}\right\}}\right\} \\
=\frac{\beta \mathbb{E}\|B\|^{\beta(\delta+1)}}{\beta(\delta+1)-2} .
\end{aligned}
$$

Based on the existing results above, we will add a general precise asymptotic result on the second-order complete moment convergence of $F_{n}(t)$.

Theorem 4. Assume that the real-valued function $g(x)$ satisfies the following conditions.

(A1) $g(x)$ is differentiable on the interval $[1,+\infty)$, which is nonnegative and strictly increasing to $\infty$.

(A2) The differentiable function $g^{\prime}(x)$ is nonnegative and the function $g^{\prime}(x) / g(x)$ is monotone. If $g^{\prime}(x) /$ $g(x)$ is monotone nondecreasing, we assume that $\lim _{x \rightarrow \infty}\left(g^{\prime}(x+1) g(x) / g(x+1) g^{\prime}(x)\right)=1$.

One has

$$
\lim _{\epsilon \searrow 0} \frac{1}{-\log \epsilon} \sum_{n=1}^{\infty} \frac{g^{\prime}(n)}{g(n)} \mathbb{E}\left\{\left\|F_{n}\right\|^{2} I_{\left\{\left\|F_{n}\right\| \geq \epsilon \sqrt{g(n)}\right\}}\right\}=\frac{\pi^{2}}{6} .
$$

Remark 5. The assumptions on $g(x)$ are rather mild; in fact, there are lots of functions satisfying them, such as $g(x)=$ $x^{\alpha}, g(x)=(\log x)^{\beta}$, and $g(x)=(\log \log x)^{\gamma}$ with suitable parameters $\alpha>0, \beta>0, \gamma>0$.

The main proofs are presented in the next section. Throughout the paper, $C$ denotes an absolutely positive constant whose value can be different from one place to another.

\section{The Proof}

We first give some propositions, which will play a key role in the proof of Theorem 4 .

Proposition 6. Under the assumptions of Theorem 4, one has

$$
\lim _{\epsilon \searrow 0} \epsilon^{2} \sum_{n=1}^{\infty} g^{\prime}(n) P\{\|B\| \geq \epsilon \sqrt{g(n)}\}=\mathbb{E}\|B\|^{2} .
$$

Proof. If $g^{\prime}(x)$ is monotone nonincreasing, by the assumptions of Theorem 4 , we can see that $g^{\prime}(x) P\{\|B\| \geq \epsilon \sqrt{g(n)}\}$ is also nonincreasing; thus

$$
\begin{gathered}
\int_{1}^{\infty} g^{\prime}(y) P\{\|B\| \geq \epsilon \sqrt{g(y)}\} d y \\
\leq \sum_{n=1}^{\infty} g^{\prime}(n) P\{\|B\| \geq \epsilon \sqrt{g(y)}\} \\
\leq \int_{0}^{\infty} g^{\prime}(y) P\{\|B\| \geq \epsilon \sqrt{g(y)}\} d y .
\end{gathered}
$$

If $g^{\prime}(x)$ is monotone nondecreasing, by the assumption that $\lim _{n \rightarrow \infty}\left(g^{\prime}(n+1) / g^{\prime}(n)\right)=1$, we can find that, for any $0<$ $\theta_{0}<1$, there exists a sufficient large number $N=N\left(\theta_{0}\right)$, such that $g^{\prime}(n+1) / g^{\prime}(n) \leq 1+\theta_{0}$ and $g^{\prime}(n+1) / g^{\prime}(n) \geq 1-\theta_{0}$ for all $n \geq N$. Thus we have

$$
\begin{aligned}
& \frac{1}{1+\theta_{0}} \int_{N}^{\infty} g^{\prime}(y) P\{\|B\| \geq \epsilon \sqrt{g(y)}\} d y \\
& \quad \leq \sum_{n=N}^{\infty} g^{\prime}(n) P\{\|B\| \geq \epsilon \sqrt{g(n)}\} \\
& \quad \leq \frac{1}{1-\theta_{0}} \int_{N-1}^{\infty} g^{\prime}(y) P\{\|B\| \geq \epsilon \sqrt{g(y)}\} d y .
\end{aligned}
$$

At the same time, by making a change of variables and integration by parts, for any $\theta \geq 0$, we have

$$
\begin{aligned}
& \lim _{\varepsilon \searrow 0} \varepsilon^{2} \int_{\theta}^{\infty} g^{\prime}(y) P\{\|B\| \geq \epsilon \sqrt{g(y)}\} d y \\
& =\lim _{\varepsilon \searrow 0} 2 \int_{2 \varepsilon \sqrt{g(\theta)}}^{\infty} y P(|\xi| \geq y) d y \\
& =2 \int_{0}^{\infty} y P(\|B\| \geq y) d y \\
& =\mathbb{E}\|B\|^{2} .
\end{aligned}
$$

By relations (9)-(11) and the fact that the result of the proposition will remain unchanged when we add or subtract some finite sums on the left hand of it, we can complete the proof by taking $\theta_{0} \rightarrow 0$.

Proposition 7. Under the assumptions of Theorem 4, one has

$$
\begin{aligned}
& \lim _{\epsilon \searrow 0} \epsilon^{2} \sum_{n=1}^{\infty} g^{\prime}(n)\left\{P\left(\left\|F_{n}\right\| \geq \epsilon \sqrt{g(n)}\right)-P(\|B\| \geq \epsilon \sqrt{g(n)})\right\} \\
& \quad=0 .
\end{aligned}
$$


Proof. A well known fact in Billingsley [10] reveals that the uniform empirical process converges weakly to Brownian bridge, $F_{n} \Rightarrow B$. By continuous mapping theorem, we have $\left\|F_{n}\right\| \Rightarrow\|B\|$. Thus, as $n \rightarrow \infty$,

$$
\Delta_{n}=\sup _{x \in \mathbb{R}}\left|P\left\{\left\|F_{n}\right\| \geq x\right\}-P(\|B\| \geq x)\right| \longrightarrow 0 .
$$

Let $A(\epsilon)=g^{-1}\left(M \epsilon^{-2}\right)$, where $g^{-1}(x)$ is the inverse function of $g(x)$ and $M$ is an arbitrary positive number; then there exists a positive constant $C$ such that

$$
\sum_{n \leq A(\epsilon)} g^{\prime}(n) \leq C \int_{1}^{A(\epsilon)} g^{\prime}(x) d x \leq C g(A(\epsilon))=C M \epsilon^{-2}
$$

thus

$$
\epsilon^{2} \sum_{n \leq A(\epsilon)} g^{\prime}(n) \leq C<\infty .
$$

By Toeplitz Lemma listed in Appendix, we know

$$
\begin{gathered}
\lim _{\epsilon \searrow 0} \epsilon^{2} \sum_{n \leq A(\epsilon)} g^{\prime}(n)\left\{P\left(\left\|F_{n}\right\| \geq \epsilon \sqrt{g(n)}\right)\right. \\
-P(\|B\| \geq \epsilon \sqrt{g(n)})\} \\
\leq \lim _{\epsilon \searrow 0} \epsilon^{2} \sum_{n \leq A(\epsilon)} g^{\prime}(n) \Delta_{n}=0 .
\end{gathered}
$$
have

$$
\begin{aligned}
& \epsilon^{2} \sum_{n>A(\epsilon)} g^{\prime}(n) P\left\{\left\|F_{n}\right\| \geq \epsilon \sqrt{g(n)}\right\} \\
& \leq C \epsilon^{2} \int_{A(\epsilon)}^{\infty} g^{\prime}(x) P\left\{\left\|F_{n}\right\| \geq \epsilon \sqrt{g(x)}\right\} d x \\
& \quad \leq C \int_{\sqrt{M}}^{\infty} y P\left\{\left\|F_{n}\right\| \geq y\right\} d y .
\end{aligned}
$$

By the result of Kiefer and Wolfowitz [11], there exists $c_{0}>0$, such that

$$
P\left\{\left\|F_{n}\right\| \geq y\right\} \leq C e^{-c_{0} y^{2}} .
$$

Then, by letting $\epsilon \searrow 0$ and then $M \rightarrow \infty$, we can get

$$
\begin{aligned}
& \lim _{M \rightarrow \infty} \limsup _{\epsilon \downarrow 0} \epsilon^{2} \sum_{n>A(\epsilon)} g^{\prime}(n) P\left\{\left\|F_{n}\right\| \geq \epsilon \sqrt{g(n)}\right\} \\
& \leq \lim _{M \rightarrow \infty} C \int_{\sqrt{M}}^{\infty} y e^{-c_{0} y^{2}} d y=0 .
\end{aligned}
$$

By Lemma 2.1 in Zhang and Yang [7], for any $x \in \mathbb{R}$,

$$
P\{\|B\| \geq x\} \leq 2 e^{-2 x^{2}} .
$$

Then, a similar argument in (19) can deduce that

$$
\lim _{M \rightarrow \infty} \limsup _{\epsilon \searrow 0} \epsilon^{2} \sum_{n>A(\epsilon)} g^{\prime}(n) P\{\|B\| \geq \epsilon \sqrt{g(n)}\}=0 .
$$

By combining (16), (19), and (21) and using the triangular inequality, we can complete the proof.
Proposition 8. Under the assumptions of Theorem 4, one has

$$
\lim _{\epsilon \searrow 0} \frac{1}{-\log \epsilon} \sum_{n=1}^{\infty} \frac{g^{\prime}(n)}{g(n)} \int_{\epsilon \sqrt{g(n)}}^{\infty} x P(\|B\| \geq x) d x=\mathbb{E}\|B\|^{2}
$$

Proof. Similar to the argument in Proposition 6, no matter whether the function $g^{\prime}(x) / g(x)$ is monotone nonincreasing or monotone nondecreasing, we can deduce the following relations by applying the change of variables and the L'Hôpital's rule:

$$
\begin{aligned}
\lim _{\epsilon \searrow 0} & \frac{1}{-\log \epsilon} \sum_{n=1}^{\infty} \frac{g^{\prime}(n)}{g(n)} \int_{\epsilon \sqrt{g(n)}}^{\infty} x P(\|B\| \geq x) d x \\
= & \lim _{\epsilon \searrow 0} \frac{1}{-\log \epsilon} \int_{1}^{\infty} \frac{g^{\prime}(x)}{g(x)} \int_{\epsilon \sqrt{g(x)}}^{\infty} t P(\|B\| \geq t) d t d x \\
= & \lim _{\epsilon \searrow 0} \frac{2}{-\log \epsilon} \int_{\epsilon \sqrt{g(1)}}^{\infty} \frac{1}{y} \int_{y}^{\infty} t P(\|B\| \geq t) d t d y \\
= & \int_{0}^{\infty} 2 t P(\|B\| \geq t) d t \\
= & \mathbb{E}\|B\|^{2} .
\end{aligned}
$$

Thus, the proof is completed.

Proposition 9. Under the assumptions of Theorem 4, one has

$$
\begin{aligned}
\lim _{\epsilon \searrow 0} \frac{1}{-} & \log \epsilon \\
\quad & \sum_{n=1}^{\infty} \frac{g^{\prime}(n)}{g(n)} \\
\quad & \int_{\epsilon \sqrt{g(n)}}^{\infty} x\left[P(\|B\| \geq x)-P\left(\left\|F_{n}\right\| \geq x\right)\right] d x=0 .
\end{aligned}
$$

Proof. If we denote $\widetilde{A}(\epsilon)=g^{-1}\left(\epsilon^{-2}\right)$, where $g^{-1}(x)$ is the inverse function of $g(x)$, then we can write

$$
\begin{aligned}
& \sum_{n=1}^{\infty} \frac{g^{\prime}(n)}{g(n)} \int_{\epsilon \sqrt{g(n)}}^{\infty} x\left[P(\|B\| \geq x)-P\left(\left\|F_{n}\right\| \geq x\right)\right] d x \\
& \leq \sum_{n \leq \widetilde{A}(\epsilon)} \frac{g^{\prime}(n)}{g(n)} \int_{\epsilon \sqrt{g(n)}}^{\infty} x\left|P(\|B\| \geq x)-P\left(\left\|F_{n}\right\| \geq x\right)\right| d x \\
& \quad+\sum_{n>\widetilde{A}(\epsilon)} \frac{g^{\prime}(n)}{g(n)} \int_{\epsilon \sqrt{g(n)}}^{\infty} x P(\|B\| \geq x) d x \\
& \quad+\sum_{n>\widetilde{A}(\epsilon)} \frac{g^{\prime}(n)}{g(n)} \int_{\epsilon \sqrt{g(n)}}^{\infty} x P\left(\left\|F_{n}\right\| \geq x\right) d x \\
& :=J_{1}+J_{2}+J_{3} .
\end{aligned}
$$


For the term $J_{1}$, via the change of variable $x=y+\epsilon \sqrt{g(n)}$, we have

$$
\begin{gathered}
\int_{\epsilon \sqrt{g(n)}}^{\infty} x\left|P(\|B\| \geq x)-P\left(\left\|F_{n}\right\| \geq x\right)\right| d x \\
=\int_{0}^{\infty}(y+\epsilon \sqrt{g(n)}) \mid P(\|B\| \geq y+\epsilon \sqrt{g(n)}) \\
\quad-P\left(\left\|F_{n}\right\| \geq y+\epsilon \sqrt{g(n)}\right) \mid d y \\
\leq\left(\Delta_{n 1}+\Delta_{n 2}+\Delta_{n 3}\right),
\end{gathered}
$$

where

$$
\begin{aligned}
& \Delta_{n 1} \\
& =\int_{0}^{\Delta_{n}^{-1 / 4}}(y+\epsilon \sqrt{g(n)}) \mid P(\|B\| \geq y+\epsilon \sqrt{g(n)}) \\
& -P\left(\left\|F_{n}\right\| \geq y+\epsilon \sqrt{g(n)}\right) \mid d y, \\
& \Delta_{n 2}=\int_{\Delta_{n}^{-1 / 4}}^{\infty}(y+\epsilon \sqrt{g(n)}) P\left(\left\|F_{n}\right\| \geq y+\epsilon \sqrt{g(n)}\right) d y, \\
& \Delta_{n 3}=\int_{\Delta_{n}^{-1 / 4}}^{\infty}(y+\epsilon \sqrt{g(n)}) P(\|B\| \geq y+\epsilon \sqrt{g(n)}) d y,
\end{aligned}
$$

and $\Delta_{n}$ is defined by (13).

Since $n \leq \widetilde{A}(\epsilon)$ implies $\epsilon \sqrt{g(n)} \leq 1$, then we can see that

$$
\begin{aligned}
\Delta_{n 1} & \leq \Delta_{n}\left(\Delta_{n}^{-1 / 4}+\epsilon \sqrt{g(n)}\right) \Delta_{n}^{-1 / 4} \\
& =\Delta_{n}^{1 / 2}+\Delta_{n}^{3 / 4} \longrightarrow 0 \quad \text { as } n \longrightarrow \infty
\end{aligned}
$$

By relation (18), when $n \rightarrow \infty$, we have

$$
\Delta_{n 2} \leq C \int_{\Delta_{n}^{-1 / 4}}^{\infty} y e^{-c_{0} y^{2}} d y \longrightarrow 0
$$
that

By using relation (20), the similar argument can prove

$$
\Delta_{n 3} \longrightarrow 0 \quad \text { as } n \longrightarrow \infty
$$

Note that $\sum_{n=1}^{\widetilde{A}(\epsilon)}\left(g^{\prime}(n) / g(n)\right) \leq C \int_{0}^{\widetilde{A}(\epsilon)}\left(g^{\prime}(x) / g(x) d x\right) \leq$ $-C \log \epsilon$. A combination of (28)-(30) and Toeplitz Lemma can lead to that

$$
\lim _{\epsilon \searrow 0} \frac{1}{-\log \epsilon} \sum_{n \leq \widetilde{A}(\epsilon)} \frac{g^{\prime}(n)}{g(n)}\left(\Delta_{n 1}+\Delta_{n 2}+\Delta_{n 3}\right)=0
$$

which indicates that

$$
\lim _{\epsilon \searrow 0} \frac{1}{-\log \epsilon} J_{1}=0 .
$$

For the term $J_{2}$, using relation (18) again, the same argument in Proposition 8 can deduce that

$$
\begin{aligned}
& \sum_{n>\widetilde{A}(\epsilon)} \frac{g^{\prime}(n)}{g(n)} \int_{\epsilon \sqrt{g(n)}}^{\infty} x P\left(\left\|F_{n}\right\| \geq x\right) d x \\
& \quad \leq C \int_{\widetilde{A}(\epsilon)}^{\infty} \frac{g^{\prime}(y)}{g(y)} \int_{\epsilon}^{\infty} \sqrt{g(y)} x P\left(\left\|F_{n}\right\| \geq x\right) d x d y \\
& \leq C \int_{1}^{\infty} \frac{1}{t} \int_{t}^{\infty} x P\left(\left\|F_{n}\right\| \geq x\right) d x d t \\
& \quad \leq C \int_{1}^{\infty} x \log x e^{-c_{0} x^{2}} d x<\infty,
\end{aligned}
$$

which implies that

$$
\lim _{\epsilon \searrow 0} \frac{1}{-\log \epsilon} J_{2}=0
$$

At last, by using relation (20) again, the similar argument as in the discussion on $J_{2}$ can yield that

$$
\lim _{\epsilon \searrow 0} \frac{1}{-\log \epsilon} J_{3}=0 \text {. }
$$
proof

Combining (25) and (32)-(35), we can complete the

Proof of Theorem 4. According to the fact that, for any random variable $\zeta$ and $a \in \mathbb{R}$,

$$
\mathbb{E} \zeta I_{\{\zeta \geq a\}}=a P(\zeta \geq a)+\int_{a}^{\infty} P(\zeta \geq x) d x
$$

we have

$$
\begin{aligned}
\mathbb{E}\left\{\left\|F_{n}\right\|^{2} I_{\left\{\left\|F_{n}\right\| \geq \epsilon \sqrt{g(n)}\right\}}\right\} & \\
= & \epsilon^{2} g(n) P\left(\left\|F_{n}\right\| \geq \epsilon \sqrt{g(n)}\right) \\
& +\int_{\epsilon \sqrt{g(n)}}^{\infty} 2 x P\left(\left\|F_{n}\right\| \geq x\right) d x .
\end{aligned}
$$

By Propositions 6 and 7, we know

$$
\lim _{\epsilon \searrow 0} \epsilon^{2} \sum_{n=1}^{\infty} g^{\prime}(n) P\left(\left\|F_{n}\right\| \geq \epsilon \sqrt{g(n)}\right)=\mathbb{E}\|B\|^{2},
$$

which shows that

$$
\lim _{\epsilon \searrow 0} \frac{1}{-\log \epsilon} \epsilon^{2} \sum_{n=1}^{\infty} \frac{g^{\prime}(n)}{g(n)} g(n) P\left(\left\|F_{n}\right\| \geq \epsilon \sqrt{g(n)}\right)=0 .
$$

By Propositions 8 and 9, we can get that

$$
\lim _{\epsilon \searrow 0} \frac{1}{-\log \epsilon} \sum_{n=1}^{\infty} \frac{g^{\prime}(n)}{g(n)} \int_{\epsilon \sqrt{g(n)}}^{\infty} x P\left(\left\|F_{n}\right\| \geq x\right) d x=\mathbb{E}\|B\|^{2} .
$$


A final combination of the above two relations can reveal that

$$
\lim _{\epsilon \searrow 0} \frac{1}{-\log \epsilon} \sum_{n=1}^{\infty} \frac{g^{\prime}(n)}{g(n)} E\left\{\left\|F_{n}\right\|^{2} I_{\left\{\left\|F_{n}\right\| \geq \epsilon \sqrt{g(n)}\right\}}\right\}=2 \mathbb{E}\|B\|^{2} .
$$

From Csörgö and Révèsz [12], we know $P(\|B\| \geq x)=$ $2 \sum_{k=1}^{\infty}(-1)^{k+1} e^{-2 k^{2} x^{2}}, x>0$, which yields that

$$
\mathbb{E}\|B\|^{2}=2 \int_{0}^{\infty} x P(\|B\| \geq x) d x=\sum_{k=1}^{\infty}(-1)^{k+1} k^{-2}=\frac{\pi^{2}}{12} .
$$

This concludes the proof of Theorem 4 .

\section{Appendix}

Lemma A.1 (Toeplitz Lemma in Loève [13]). Let $a_{n k}, k=$ $1,2, \ldots, k_{n}$, be numbers such that, for every fixed $k, a_{n k} \rightarrow 0$, and for all $n, \sum_{k}\left|a_{n k}\right| \leq c<\infty$. If $x_{n} \rightarrow 0$, then

$$
x_{n}^{\prime}=\sum_{k} a_{n k} x_{k} \longrightarrow 0 .
$$

In particular, if $b_{n}=\sum_{k=1}^{n} a_{k} \uparrow \infty$ and $x_{n} \rightarrow 0$, then

$$
\frac{1}{b_{n}} \sum_{k=1}^{n} a_{k} x_{k} \longrightarrow x
$$

\section{Conflict of Interests}

The authors declare that there is no conflict of interests regarding the publication of this paper.

\section{Acknowledgments}

The work is supported by the National Natural Science Foundation of China (nos. 11326173 and 11401169) and Foundation of Henan Educational Committee (nos. 13A110087 and 2014JSJYYB-011). The authors would like to thank the referee for some valuable comments and suggestions.

\section{References}

[1] P. L. Hsu and H. Robbins, "Complete convergence and the law of large numbers," Proceedings of the National Academy of Sciences of the United States of America, vol. 33, pp. 25-31, 1947.

[2] L. E. Baum and M. Katz, "Convergence rates in the law of large numbers," Transactions of the American Mathematical Society, vol. 120, pp. 108-123, 1965.

[3] C. C. Heyde, "A supplement to the strong law of large numbers," Journal of Applied Probability, vol. 12, pp. 173-175, 1975.

[4] Y. S. Chow, "On the rate of moment convergence of sample sums and extremes," Bulletin of the Institute of Mathematics: Academia Sinica, vol. 16, no. 3, pp. 177-201, 1988.

[5] W. Liu and Z. Lin, "Precise asymptotics for a new kind of complete moment convergence," Statistics \& Probability Letters, vol. 76, no. 16, pp. 1787-1799, 2006.
[6] A. Gut and J. Steinebach, "Precise asymptotics-a general approach," Acta Mathematica Hungarica, vol. 138, no. 4, pp. 365-385, 2013.

[7] Y. Zhang and X. Yang, "Precise asymptotics in the law of the iterated logarithm and the complete convergence for uniform empirical process," Statistics \& Probability Letters, vol. 78, no. 9, pp. 1051-1055, 2008.

[8] Q. P. Zang and W. Huang, "A general law of moment convergence rates for uniform empirical process," Acta Mathematica Sinica, vol. 27, no. 10, pp. 1941-1948, 2011.

[9] Y. Chen and L. Zhang, "Second moment convergence rates for uniform empirical processes," Journal of Inequalities and Applications, vol. 2010, Article ID 972324, 2010.

[10] P. Billingsley, Convergence of Probability Measures, John Wiley \& Sons, New York, NY, USA, 1968.

[11] J. Kiefer and J. Wolfowitz, "On the deviations of the empiric distribution function of vector chance variables," Transactions of the American Mathematical Society, vol. 87, pp. 173-186, 1958.

[12] M. Csörgö and P. Révèsz, Strong Approximations in Probability and Statistics, Academic Press, New York, NY, USA, 1981.

[13] M. Loève, Probability Theory, Springer, New York, NY, USA, 4th edition, 1977. 


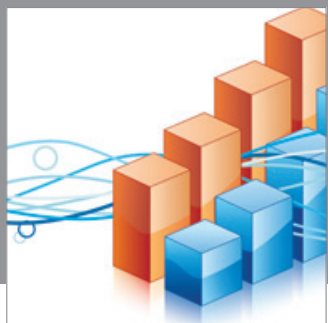

Advances in

Operations Research

mansans

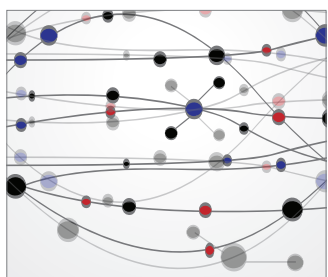

The Scientific World Journal
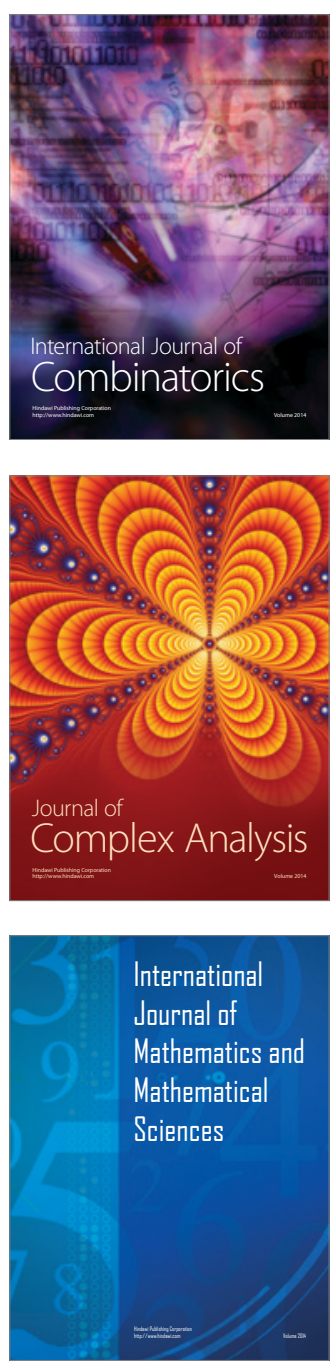
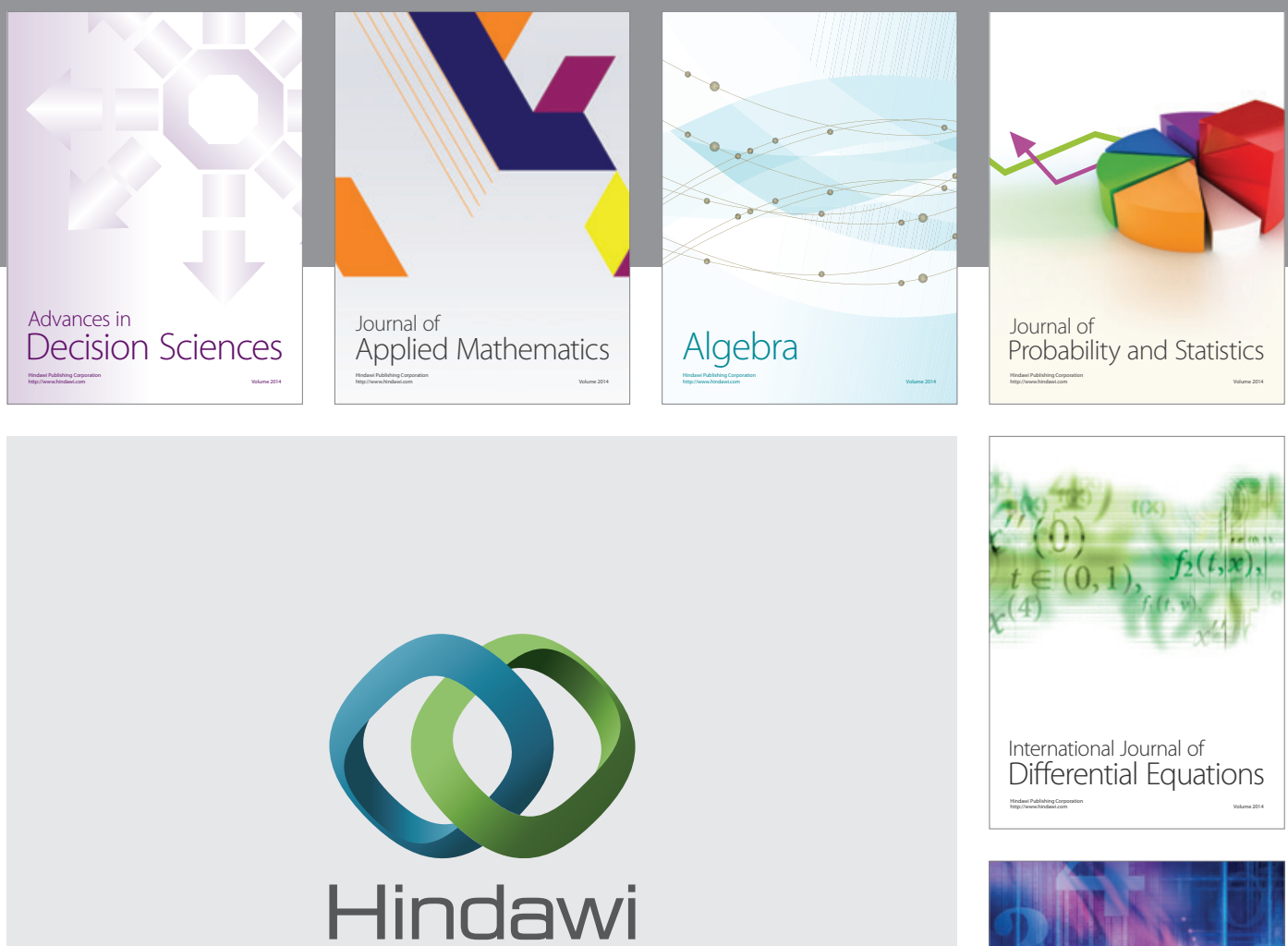

Submit your manuscripts at http://www.hindawi.com
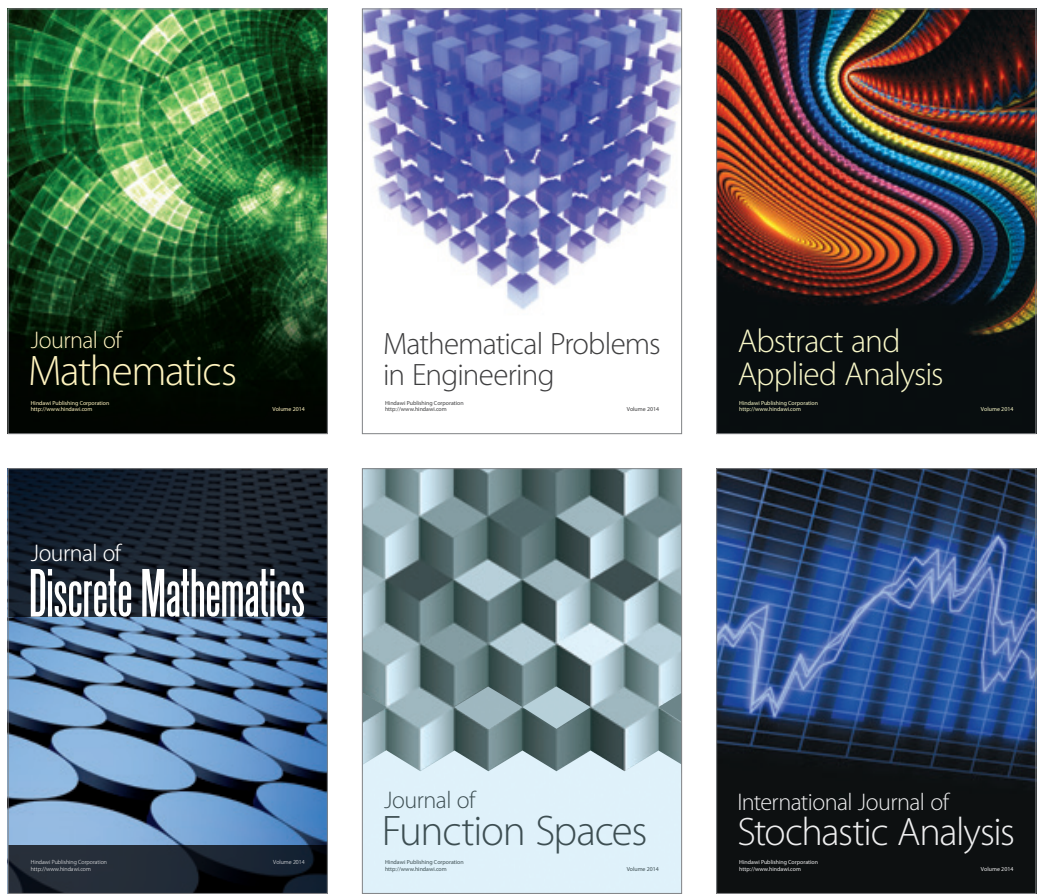

Journal of

Function Spaces

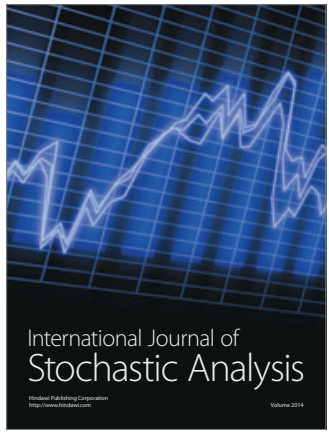

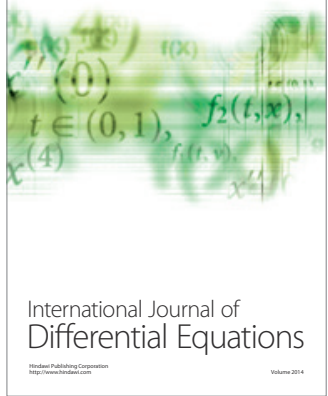
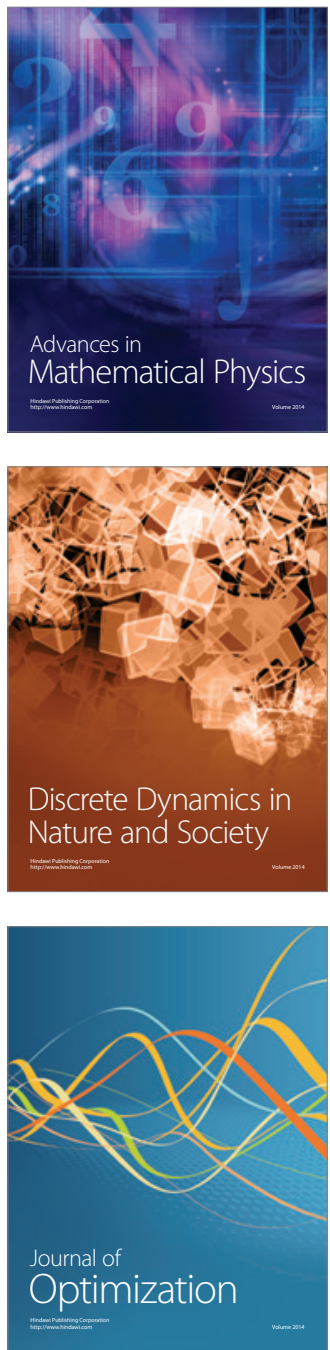